\section{Introducing the PeaceKeeping Operations Corpus (PKOC)}

\author{
Elio Amicarelli
}

Independent Researcher

\author{
Jessica Di Salvatore $\mathbb{B}$
}

Department of Politics and International Studies, University of Warwick
Journal of Peace Research

$1-12$

(C) The Author(s) 2021

(c) (1) (8)

Article reuse guidelines:

sagepub.com/journals-permissions

DOI: $10.1177 / 0022343320978693$

journals.sagepub.com/home/jpr

@SAGE

\begin{abstract}
Scholars have used United Nations Secretary-General's (UNSG) reports to extract information on peacekeeping operations (PKOs). As key peacekeeping political documents, UNSG reports contain much more information on the politics of peacekeeping. Furthermore, manually extracting information is costly and time-consuming. By providing a machine-readable collection of the UN Secretary-General's Reports on PKOs (1994-2020), the PeaceKeeping Operations Corpus (PKOC) offers highly structured and multiformat text data that connect the peace and conflict research community to recent advancements in text-as-data techniques. Besides paving the way for the first quantitative content analyses on PKOs, PKOC speeds up and expands the range of information analysable from these documents and allows researchers to query them in a quicker, systematic and reproducible way. In this article, we discuss PKOC's core characteristics. As illustration of the innovative potential of PKOC, we show how text-as-data approaches provide more nuanced understanding on PKOs' evolution toward multidimensionality, both over time and within missions. While last generation PKOs are assumed to be multidimensional, we show how they vary in multidimensionality and how their complexity also changes throughout their life-cycle.
\end{abstract}

\title{
Keywords
}

peacekeeping, quantitative text analysis, text-as-data

\section{Introduction}

The UN produces few statistics on peacekeeping operations (PKOs), ${ }^{1}$ but plenty of documents that talk about them. Little effort has been made, however, in using and analysing reports from the UN Secretary-General (UNSG) on PKOs as text data. To this aim, this article introduces the PeaceKeeping Operations Corpus (PKOC), a digitized, structured collection of all UNSG reports on PKOs from 1994 to 2020. In the quantitative literature on peacekeeping, these reports are widely used to extract information on missions' activities, cooperation with the government and location of blue helmets

\footnotetext{
${ }^{1}$ These are referred to as peace operations, particularly after the Department of Peacekeeping Operations became Department of Peace Operation in 2019.
}

(Clayton et al., 2017). We propose to use these documents to shed light on political and strategic aspects of PKOs.

PKOC enables and expands the spectrum of the UNSG reports' usage in peacekeeping research along three main dimensions. First, PKOC enables agile interrogation of a relatively large corpus at the researchers' fingertips. For example, to evaluate research feasibility by checking whether a piece of information is contained in the corpus, it is not necessary to download manually and then read hundreds of documents; as any other digital text, PKOC allows researchers to quickly query the entire body of UNSG reports (1994-2020). Second,

\footnotetext{
Corresponding author: jessica.di-salvatore@warwick.ac.uk
} 
PKOC can be used to explore the dynamics of peace operations and test hypotheses by extracting relevant information at the mission-report level. For example, one can test whether different degrees of prioritization of civilians' protection enable the mission to save more lives. Notably, the corpus makes the task of variable creation faster and, more importantly, reproducible.

The third and most innovative contribution of PKOC is that it represents an entry point for conflict and peace research community into quantitative text analysis. Quantitative content analysis treats words and language as data themselves, and performs statistical analysis on large collections of texts. Other research fields in the Social Sciences have benefited from the text-as-data wave, particularly with the development of Natural Language Processing functionalities made available for most used statistical software (e.g. quanteda package for R by Benoit et al. (2018)). PKOC connects the peacekeeping research community to these exciting trends in quantitative political science (Benoit et al., 2016; Grimmer \& Stewart, 2013). Within extant peacekeeping studies, analysing UNSG reports as political texts allows the tracing of the development of important narratives and discourses around peacekeeping as a policy tool. Hence, PKOC paves the way to novel theoretical insights regarding the UN decision-making process (e.g. how does UNSG reporting influence decisions to extend or adjust mandates?), missions' bias (e.g. does UN reporting of civilian victimization vary by perpetrator?) and institutionalization of norms in international organizations (e.g. how and when do gender issues enter the peacekeeping discourse?). This article focuses on quantitative uses of the corpus but it is worth pointing out that the analysis of corpora is suited for qualitative analysis as well, particularly discourse analysis (Baker, 2006).

In the next section, we briefly review existing data on PKOs relying on the coding of UNSG reports. Then we introduce PKOC and describe the structure and main features of the corpus in Section 3. In Section 4, we explore the evolution of peacekeeping by comparing trends in reporting and multidimensionality over time and, more importantly, across missions. We propose new, more fine-grained measures of missions' features, and use UNMIK in Kosovo as a case study to explore within-mission variation in multidimensionality.

\section{Data on peacekeeping operations}

The growing academic interest in peacekeeping has been accompanied by a move from measuring presence or absence of peacekeeping missions in a given conflict-year to information on the timing, location, personnel type and activities they carry out. The International Peace Institute (IPI) has compiled data on missions' composition and contributions. ${ }^{2}$ In the Geo-PKO project, Cil et al. (2019) estimate the number of peacekeepers deployed subnationally using maps from UNSG reports. Data on UN peacekeeping missions' leadership have also become available (Bove et al., 2020). Two additional sets of data contribute to the disaggregation trend we currently see in the peacekeeping literature. The PKO Location Event Data (PKOLED) is an event-based dataset that codes cooperative and conflictual events involving the UN mission in the host country either as actor or target (Dorussen \& Ruggeri, 2007). Finally, the PKO Governance (PKOGOV) dataset codes events involving both UN peacekeepers and the host government (Dorussen \& Gizelis, 2013). As for Geo-PKO, PKOLED and PKOGOV data use UNSG reports as key source. To our knowledge, there are three more ongoing data collections using UNSG reports, namely by Smidt on peacekeeping activities during elections (Smidt, forthcoming), by Hultman on activities related to protection of civilians (see Clayton et al., 2017 for details), and by Kjeksrud (2019) on the use of violence for civilians' protection.

The reliance on UNSG reports, however, has some shortcomings that are worth pointing out. Reports aim at informing the UN Security Council (UNSC) about some key developments in the host country and policy areas UN troops focus on. However, not only is there variation in the number of reports published each year for each mission, but also the richness and accuracy of reporting vary. After all, UNSG reports are political documents, and may suffer from bias in reporting specific events or developments on the ground. One of the aims of PKOC is analysing UNSG reports as political and strategic rather than operational documents with semantic and linguistic features that reveal more about the politics of peacekeeping. Reports are not expected to be comprehensive accounts of specific events in the field; the purpose of UNSG reports is to inform the UNSC about the overall situation, not to provide the exact account of missions' activities. We provide an overview of what UNSG reports are, what they contain, how they are drafted and the reporting line from the field to the Headquarters (see Online appendix A.3).

\footnotetext{
${ }^{2}$ Available at: https://www.ipinst.org/providing-for-peacekeepingdatabase.
} 
UNSG reports in PKOC by year
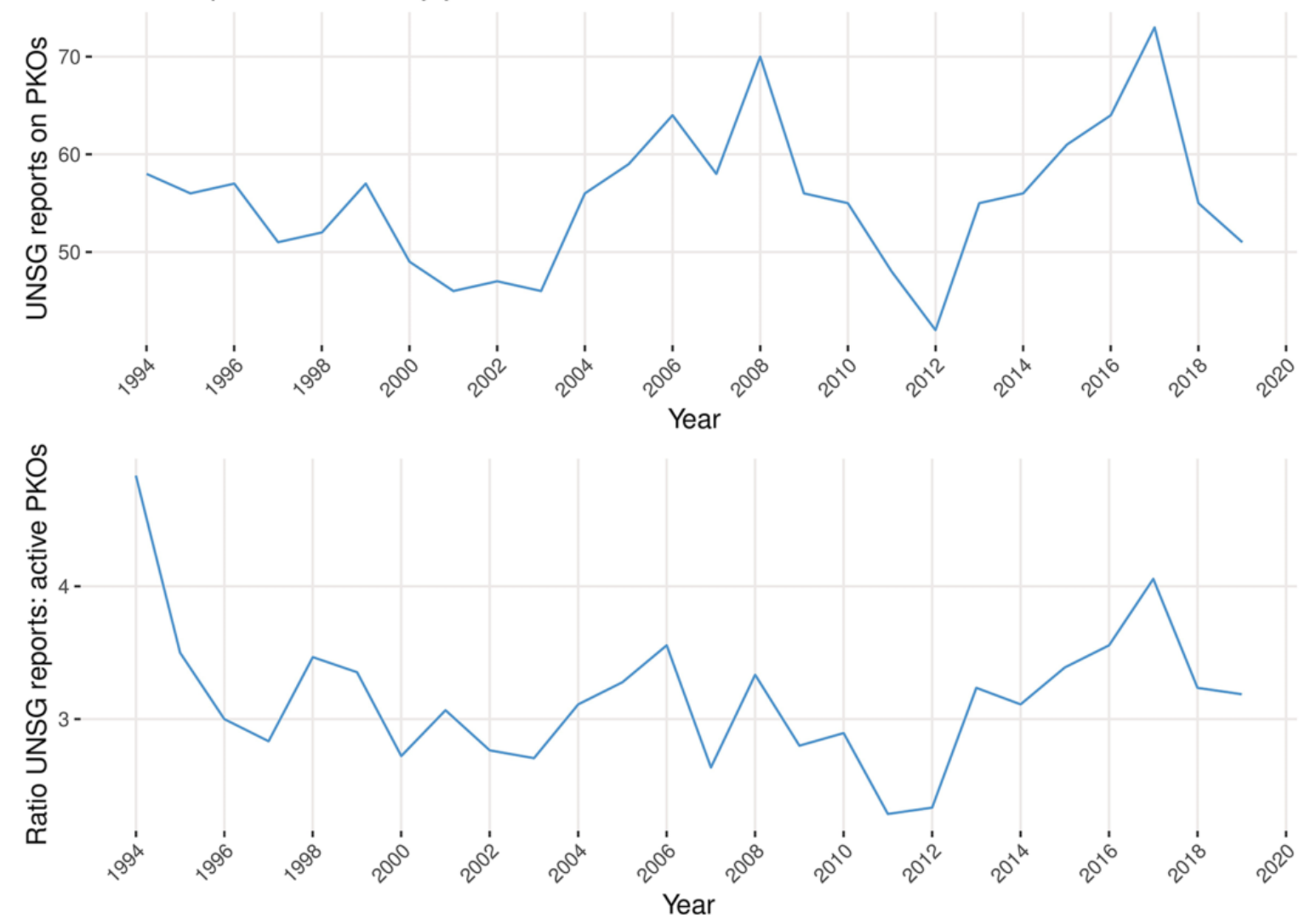

Figure 1. Number of reports by year

\section{PeaceKeeping Operations Corpus (PKOC)}

\section{Overview}

PKOC is a collection of textual data on peacekeeping retrieved from the UNSG reports. It includes 1,455 reports covering 68 missions from 1994 to 2020. Of these 68 missions, 61 have complete coverage in the corpus since they started after $1994 .^{3}$ The corpus can be regularly updated via an automated pipeline that downloads new reports from the UNSG webpage, processes, and adds them to the existing corpus. ${ }^{4}$ Figure 1 shows the number of UNSG reports included in the corpus by year, as absolute number (top) and as ratio relative to active missions (bottom). Excluding 2020 (ongoing), the number of reports varies from a minimum of 42 in 2012 to a maximum of 73 in 2017. Despite some fluctuations over time, no clear time trend emerges

\footnotetext{
${ }^{3}$ See list in Online appendix A.1.

${ }^{4}$ Since the UN does not have an API (Application Programming Interface) to query the documents, the procedure requires a human-in-the-loop approach to check each step of the automated pipeline.
}

in the overall reporting activity, even when adjusting by the number of active missions in each year.

While the yearly number of reports has not changed significantly, their length might have increased as missions have become broader in scope. Figure 2 displays the distribution of yearly reports' word count. The yearly mean number of tokens (i.e. list of unique words) more than doubled (approximately from 3,000 in 1994 to 9,700 in 2019). Thus PKOs' reporting has become either more extensive or more verbose (or both), possibly because multidimensional missions have more tasks to report on. Figure 3 shows the frequency of reporting for all months of deployment of PKOs since 1994. Most missions receive a good coverage, and frequency of reports is generally consistent within missions. Yet, important variation exists across missions, which may be explained by deployment phase, operational needs or UNSC oversight. For example, reports for the UNAMID (Darfur) or UNMISS (South Sudan) are frequent, probably because of the scale of violence and humanitarian crisis in the region. For data projects collecting using UNSG reports as sources, the problem of reporting frequency does not seem to be a major issue within most 


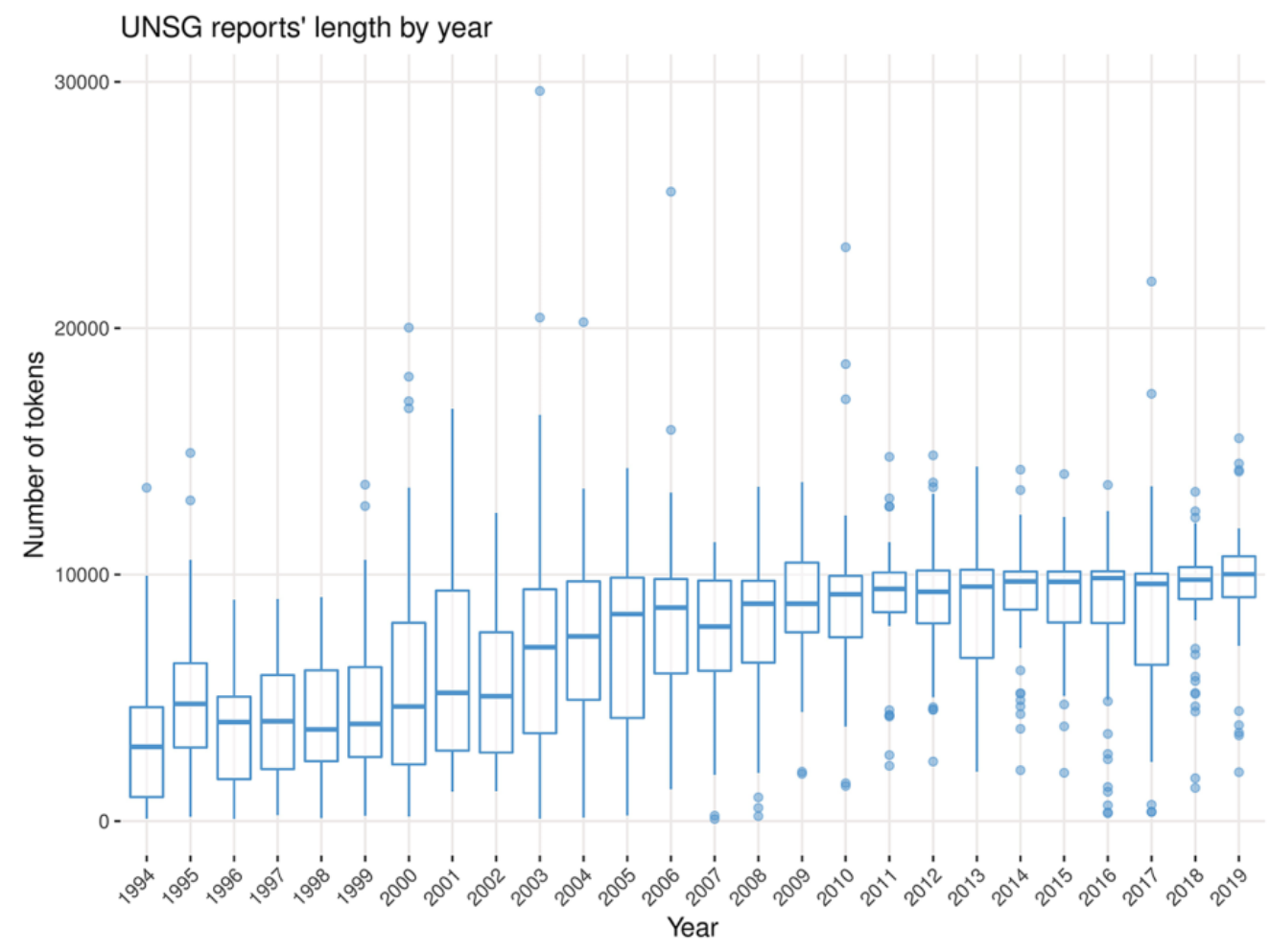

Figure 2. Distribution of UNSG reports' length by year.

Boxplots show yearly interquartile ranges and medians. Circles are values $>1.5$ times the interquartile range

missions, but one should be careful when comparing missions to each other.

Next, we provide information on PKOC's structure and content. To simplify the use of PKOC, we have designed it as a highly structured corpus. This means that the documents in PKOC have a rich indexing structure that supports the formulation of ad hoc retrieval tasks. In other words, structured corpora are easier to query thanks to their metadata, a key feature that distinguishes a corpus from a simple archive. In addition, PKOC comes in three different formats and each of them can be seen as a platform that makes certain tasks or types of analysis easier to perform. The structured and multiformat nature of PKOC is described in the next subsections.

\section{Structure}

Digital collections of documents differ on the level of meta-information associated with the documents they are made of. Without documents' meta-information, it would be difficult to retrieve specific texts from a corpus according with a set of desired criteria. PKOC's indexing structure provides meta-information at the report level on several dimensions: mission acronym, report code, period of deployment, month, year and host country code. Indexes can be combined so that users can easily subset the data according to different needs. If someone is interested in exploring how a pre-specified set of missions has evolved over time, they could leverage the mission name and month-year indexes to quickly extract only the relevant documents from the corpus and order them over time. Or, if interested in comparing missions in a specific deployment phase, it is possible to retrieve information by subsetting the corpus using the periodof-deployment index. Since each document in PKOC is linked to country codes, years, months and mission indexes, it can be easily linked to existing peacekeeping and conflict data using the same identifiers.

\section{Formats}

When considered as data, text is a ductile resource. It can be used for different types of analysis that require specific pre-processing intermediate steps. For example, researchers interested in discovering topics in documents, usually need to prepare the data such that uninformative words are stripped out from the corpus and to reduce similar words to a common root (this latter procedure is called stemming, Manning, Raghavan \& Schutze, 2008). Alternatively, to investigate relationships among actors or extract events from documents, words must be 
UNSG reports coverage of PKOs by month

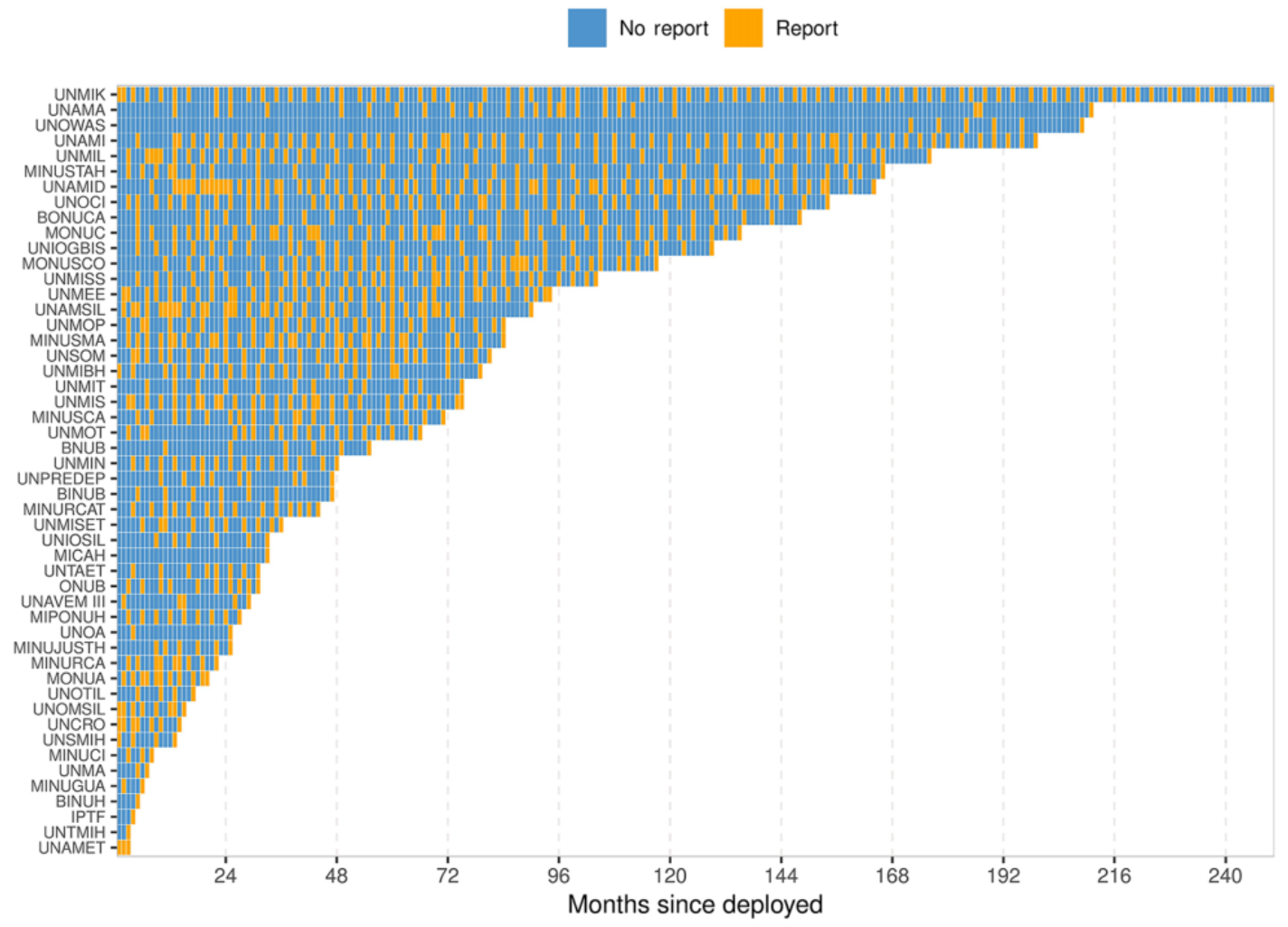

Figure 3. UNSG reporting based on PKOC

associated with classes such as nouns, verbs and adjectives. To support different types of analysis, PKOC comes in three formats. First, plain PKOC (pPKOC) is a digital version of raw UNSG reports 'as they are': pPKOC allows researchers to move across reports and to easily query them in their original format; furthermore, it offers the possibility to customize the corpus. The second format, reduced PKOC (rPKOC), contains a pre-processed version of the reports where stopwords, ${ }^{5}$ punctuation, numbers, symbols and annexes are removed, words are lowercased and stemmed. ${ }^{6}$ Many Natural Language Processing methods require text to be pre-processed (Manning, Raghavan \& Schutze, 2008), hence we provide two ready-to-use reduced versions of the corpus. In version one, only general stopwords are removed, while in version two, domainspecific stopwords are also excluded (e.g. 'United Nations', 'Security Council'). ${ }^{7}$ Finally, the tagged

\footnotetext{
${ }^{5}$ Stopwords are common words in a language or set of documents (e.g. 'the').

${ }^{6}$ Stemming was performed using the Porter stemming algorithm.

${ }^{7}$ Domain-specific stopwords are words which are common in UNSG reports, i.e. they appear in more than $98 \%$ of the documents.
}

version ( $\mathrm{tPKOC)}$ is similar to the plain one in the sense that text is complete but each word has a set of grammatical annotation and is associated with a grammatical class and with named entities. Tagging the corpus is the first step toward automatic information extraction as it allows algorithms to interpret sentences as relationships between entities and to identify actors (i.e. nouns) and actions (i.e. verbs). ${ }^{8}$ As we are not in a position to assess the accuracy of the UNSG reporting in the absence of an alternative data source to compare, we advise caution when extracting information on missions' activities from reports. As we discuss in the Online appendix (A.3), UNSG reports are more accurate to study peacekeeping on a strategic rather than operational level. For further clarification about the different characteristics of the $\mathrm{p} / \mathrm{r} / \mathrm{t}$ formats of PKOC, Table I compares the same sentence as it is represented in the three different formats. As a final note, to facilitate the interoperability of the data

\footnotetext{
${ }^{8}$ The tagging has been performed using the Stanford CoreNLP Toolkit (Manning et al., 2014). For a complete list of word classes, see Table A.2 in the Online appendix.
} 
Table I. Example of PKOC formats

\begin{tabular}{|c|c|}
\hline Format & Sentence \\
\hline Plain & $\begin{array}{l}\text { The Government of the Sudan and the Sudan } \\
\text { Peoples Liberation Movement/Army (SPLM/A) } \\
\text { agreed to share responsibility, over a period of six } \\
\text { and a half years, for creating a new model of } \\
\text { governance by restructuring the political system } \\
\text { on the principles of democracy and respect for } \\
\text { human rights; }\end{array}$ \\
\hline Reduced & $\begin{array}{l}\text { agre share respons creat govern restructur polit } \\
\text { system principl democraci respect human right }\end{array}$ \\
\hline Tagged & $\begin{array}{l}\text { The/DT Government of the Sudan/NNP and/CC } \\
\text { the/DT Sudan Peoples' Liberation Movement/ } \\
\text { NNP SPLM/NNP agreed/VBD to/TO share/VB } \\
\text { responsibility/NN,//, over/IN a/DT period/NN } \\
\text { of/IN six/CD and/CC a/DT half/NN years/ } \\
\text { NNS,/, for/IN creating/VBG a/DT new/JJ } \\
\text { model/NN of/IN governance/NN by/IN } \\
\text { restructuring/VBG the/DT political/JJ system/ } \\
\text { NN on/IN the/DT principles/NNS of/IN } \\
\text { democracy/NN and/CC respect/NN for/IN } \\
\text { human/JJ rights/NNS;/: }\end{array}$ \\
\hline
\end{tabular}

across different platforms, PKOC can provide data in different extensions, including Excel spreadsheets.

\section{The evolution of PKOs}

In this section, we use PKOC to analyse the evolution of PKOs and their multidimensionality. Recent peacekeeping missions have ambitious mandates that go beyond security related tasks and include assistance to governments in reforming institutions, holding elections, promoting human rights and fostering economic recovery. Such missions are thus multidimensional to the extent that they require more than a military presence focused on the security dimension. They are also referred to as integrated, with other UN agencies working alongside blue helmets to coordinate on development, humanitarian issues and a variety of policy domains (Fortna \& Howard, 2008).

Figure 4 gives a glance into the evolution of protection of civilians (PoC) in peacekeeping since 1994. The plot shows the percentage of sentences in all UNSG reports in a given year that contain expressions related to protection of civilians. There is a common consensus around the fact that $\mathrm{PoC}$ has become increasingly important within UN missions, and the increasing use of the key terms in UNSG reporting supports this clearly.

The first UN mission with a PoC mandate was UNAMSIL (Sierra Leone), authorized at the end of
1999. ${ }^{9}$ Indeed, mentions of PoC increase since the early $2000 s^{10}$ (with a peak in 2002), but only pick up after 2009. Unsurprisingly, in 2009 the UNSC agreed that protection activities 'must be given priority in decisions about the use of available capacity and resources, including information and intelligence resources, in the implementation of mandates' (UNSC, 2009: para. 19). The peacekeeping literature has classified missions based on whether they had a PoC mandate using a dichotomous measurement. But with the majority of ongoing PKOs having a PoC mandate, this measurement does not capture much variation across recent missions. MINUSTAH (Haiti) and MONUC (Democratic Republic of the Congo) both had PoC mandates but they implemented this task. MINUSTAH was encouraged to assist the government in protecting civilians, while MONUC's mandate was required to use all necessary means to implement PoC, with little references to assisting the government in doing so. Not to mention that the security threats to civilians in DRC and Haiti are different. Figure 5 depicts how prominent is protection of civilians in UNSG reporting by mission, to further show how tokens' occurrence highlights cross-missions' variations in PoC focus. In fact, if we compare MINUSTAH and MONUC on this measure of PoC centrality, MINUSTAH is less focused on PoC than MONUC, even though all have a mandate to protect civilians. It is not surprising to see how important is PoC for the UN mission in South Sudan (UNMISS), in the light of the current humanitarian crisis the country is facing.

Notably, UNMISS has been struggling to fulfil its PoC mandate, to the point that the former UNSG Ban $\mathrm{Ki}-\mathrm{Moon}$ warned about the possibility of ethnic cleansing escalating to genocide. ${ }^{11}$ If we assume that UNSG reports will not mention issues that missions are failing at, then we should see fewer PoC references in UNMISS reports. While tasks where blue helmets do not meet expectations set in mandates are likely underreported, the overall strategic focus of UNMISS is still evident. One reason for this, is that the UNSC may request the UNSG to report on specific aspects of the mandate. We

\footnotetext{
9 There are rare mentions of PoC before 1999, for example in UNAMIR (Assistance Mission for Rwanda) in 1994, UNOMIL (Observer Mission in Liberia) in 1995 and UNIFIL (Interim Force in Lebanon) in 1996. Being so rare, they are not visible in the line trend.

${ }^{10}$ Interestingly, and in line with trends shown, Hultman (2013) finds that the implementation of PoC as a norm by the UNSC manifested clearly only after 1999 .

${ }^{11}$ Available at: https://rb.gy/kqdiar.
} 


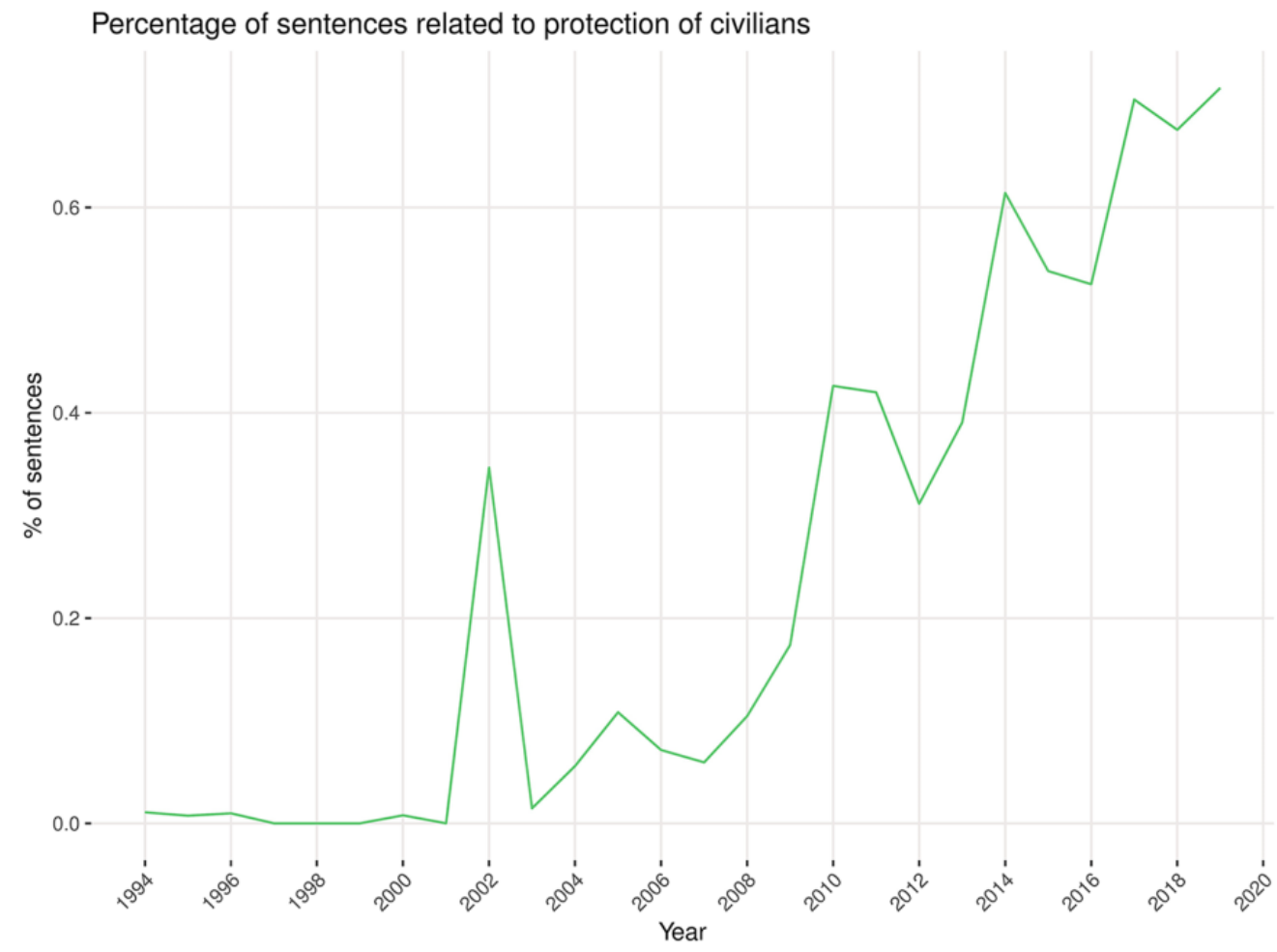

Figure 4. Percentage of sentences containing 'protection of civilians' or 'protection to civilians' or 'protecting civilians'

\section{Percentage of sentences related to protection of civilians}

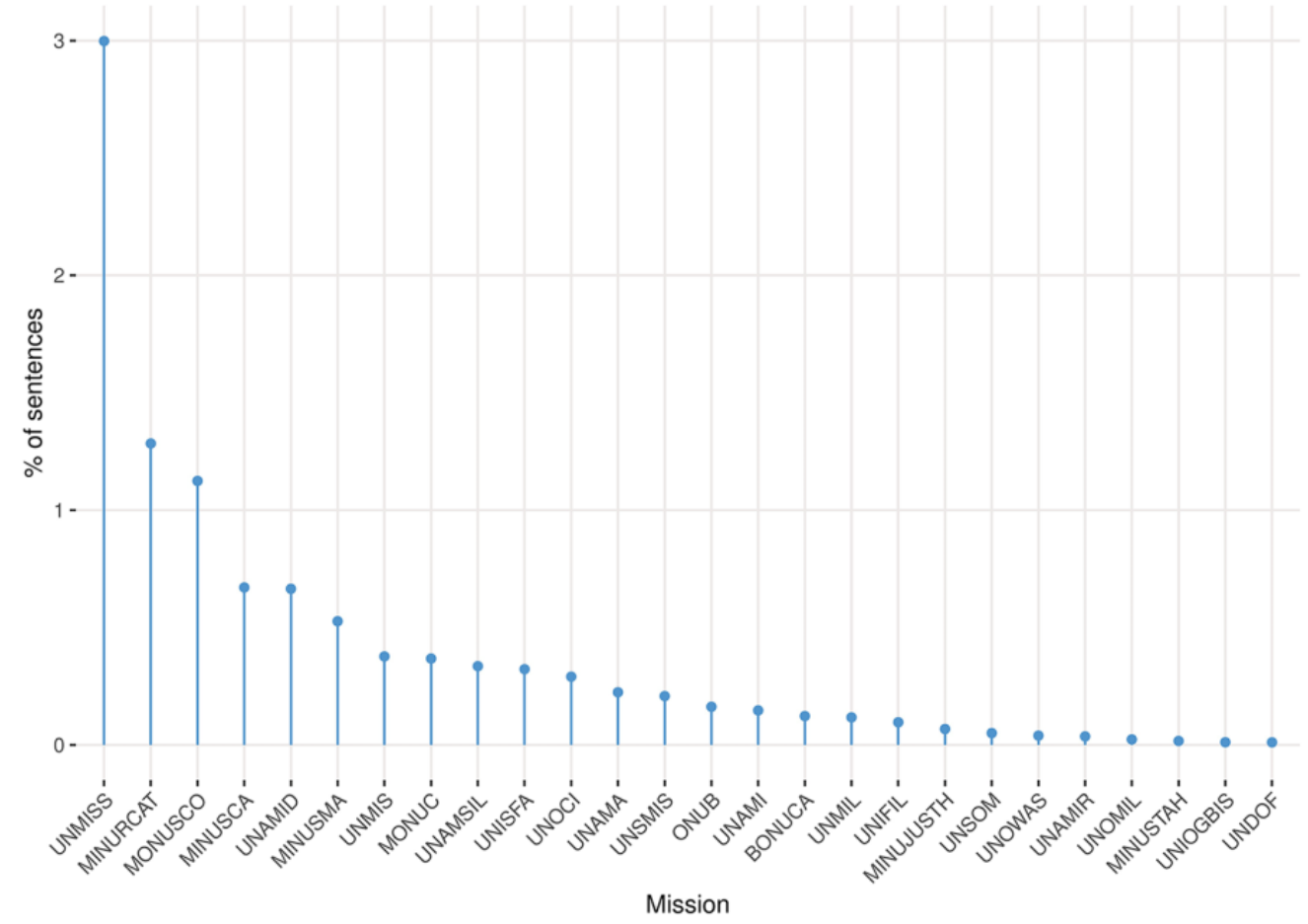

Figure 5. Percentage of sentences containing 'protection of civilians' or 'protection to civilians' or 'protecting civilians' by mission (only $\%$ above 0 included) 
would expect that the more challenges missions face on specific tasks, the more likely the UNSC will make these requests to maintain more oversight. Hence, while the UNSG will report on all policy areas strategically relevant to the mission, what will be exactly reported is a different matter, and pertains to the distinction we draw in the Online appendix regarding UNSG as strategy-oriented vs. operation-oriented documents. In the next section, we also briefly touch upon another issue concerning the relationship between the UNSC and the UNSG on peacekeeping, more specifically the relationship between UNSC mandates and UNSG reports.

This illustration points out that dummy variables for PoC mandate are not always well suited to capture variation across recent missions. We also suggest using PKOC to construct alternative, continuous measures of missions' PoC focus. Most recent missions are also often referred to as multidimensional. The progressive expansion of the domains in which UN missions operate is often discussed and taken as given, but it has never been examined from a quantitative standpoint. In the next section, we investigate this topic by capturing missions' multidimensionality via the degree of diversifications of the documents' reporting structure.

\section{Multidimensional peacekeeping? A quantitative textual analysis approach}

In this section we use PKOC for three goals that can only be achieved with text-as-data approaches. First, we propose and calculate a measure of peacekeeping multidimensionality derived from the structure of UNSG reporting on PKOs; second, we investigate how peacekeeping multidimensionality has changed over time; finally, we zoom in on one specific mission to see how multidimensionality changes within the lifespan of a UN peace mission.

UNSG reports are structured in sections that cover a specific topic summarized in a commonly used heading. In the Online appendix (A.3) we describe the recurrent structure of reports that we leverage to explore strategic developments of PKOs. Once headings are extracted from the corpus, we process them to reduce each report to a concatenation of words. The procedure is detailed in the Online appendix (A.4). Briefly, once all titles are extracted, we clean the set of titles by removing entries that do not refer to missions' activities (e.g. 'Introduction') or do not refer to a clear domain of activity (e.g. 'Status of Deployment'). The list of informative titles is then stemmed (see section on Formats). Ultimately, each section title is reduced to the minimum parts that are the most informative about the domain of blue helmets' activities. As an example, this four-step procedure would look as follows:

a. introduction; status of deployment; economic regeneration; reconstruction and development; demobilization and reintegration

b. introduction; status of deployment; economic regeneration; reconstruction and development; demobilization and reintegration

c. economic regeneration; reconstruction and development; ${ }^{12}$ demobilization and reintegration

d. econom reconstruct demobilizat reintegrat

Groupings of words represent policy domains the UNSG is reporting on. Once stemmed, grammar and ordering of these groupings are no longer informative; occurrences and multiplicity of words are maintained and used to construct an index of a mission's multidimensionality. Since ordering is not relevant, we can use a Bag of Words (BoW) model as representation of the documents in the corpus, which allows us to calculate Shannon's index of information entropy to measure documents' complexity. For each document $i$, we calculate an information entropy index $H$ to quantify the information contained in the document. This quantification considers the probability that a domain appears in the text, so that domains that are less likely to be present are much more informative when they are detected in a document. In the literature on political communication and media framing, Shannon's index is used to understand and measure media's attention diversity, 'that is, the degree to which attention on an agenda is distributed across items, from complete concentration (a single item receiving all attention) to complete diversity (all items receiving an equal level of attention)' (Boydstun, Bevan \& Thomas, 2014: 174). ${ }^{13}$

Shannon's index never takes negative values, and equals 0 when the probability of seeing a domain in a document is 1 (i.e. there is no uncertainty). Lower $H$

12 See A.4 for the explanation of why development is deleted in this case.

13 The formula for Shannon's entropy is as follows:

$$
H\left(X_{i}\right)=-\sum_{i=1}^{n} P\left(d_{i}\right) \log _{2} P\left(d_{i}\right)
$$

where $P\left(d_{i}\right)$ is the probability that a domain $d$ (e.g. 'econom') appears in reports for mission $X$; alternatively, $P\left(d_{i}\right)$ can be thought of as the proportion of total attention the domain $d$ receives in the UNSG reporting for a given mission. 


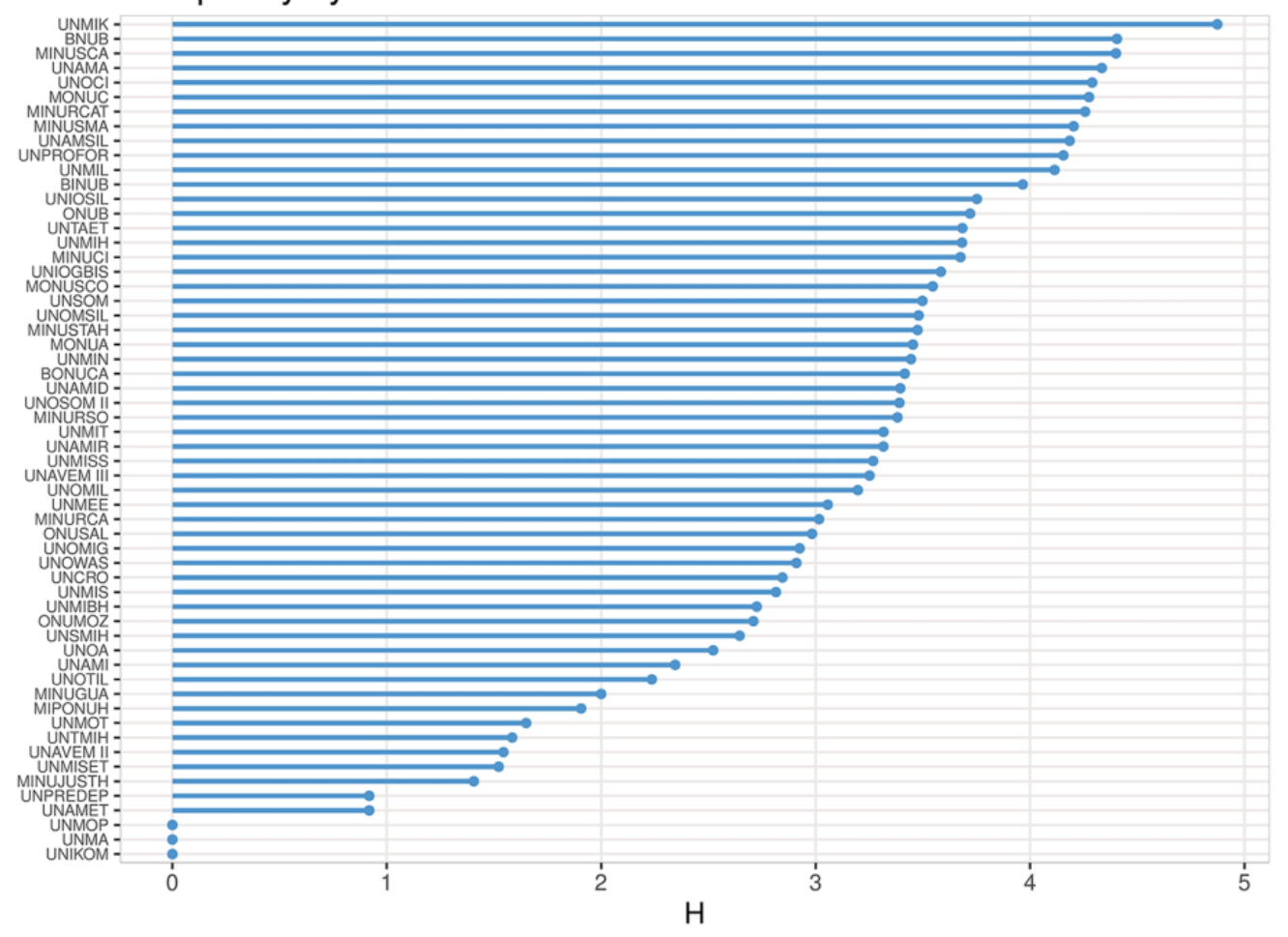

Figure 6. Information entropy by mission

among documents can also be interpreted as documents having a 'more focused message' (Munger et al., 2019: 826). We use the entropy index $H$ to (i) compare missions to each other, (ii) describe temporal trends in the evolution of peacekeeping, and (iii) explore the evolution of activities within the life-cycle of one PKO.

Figure 6 shows Shannon's information entropy for all missions that started after 1990. Missions that are commonly considered complex and multidimensional such as UNMIK (Kosovo), MINUSCA (Central African Republic), MONUC (Democratic Republic of the Congo), UNOCI (Ivory Coast), UNAMSIL (Sierra Leone), MINUSMA (Mali) and UNMIL (Liberia) do report high levels for entropy; in other words, they are more likely to report on different domains. At the bottom of the list, among others, we find UNAMET; given that this mission in East Timor was deployed to organize and verify the referendum on autonomy from Indonesia, it is clear why the mission score so low on the $H$ index. Similarly, UNPREDEP (Macedonia) or UNAVEM II (Angola) had monitoring and verification as main tasks, few domains if compared to recent, more complex operations.

To further understand how missions are classified based on the reporting, Figure 7 compares domains of activity for a relatively simple mission, UNAVEM II, and a more multidimensional mission, MINUCI. It emerges that UNAVEM II focused significantly on the humanitarian crisis in Angola, followed by political developments and the military situation. This is in line with a traditional peace operation. Interestingly, the correspondence with its mandate is not obvious. The mandate encourages support to humanitarian agencies, but the UNSC never explicitly requests it. The dire humanitarian crisis, however, explains why UNAVEM II reports focus predominantly on this issue. The inverse dynamic is also possible. UNAVEM II had a mandate to support the electoral process in Angola, which however is something that does not emerge from the reports. Indeed, in 1994, the UNSG reported that: 'Once the outstanding issues on national reconciliation are resolved, the discussions will concentrate on the conclusion of the electoral process and on the future mandate of the United Nations and the role of the three observer States' (S/1994/374), suggesting that other issues (i.e. humanitarian crisis) had to be addressed before carrying out the electoral mandate.

MINUCI's (Ivory Coast's) reporting, on the other hand, had human rights and economic development as key concerns. These domains suggest MINUCI was less military-focused than UNAVEM. Furthermore, the 

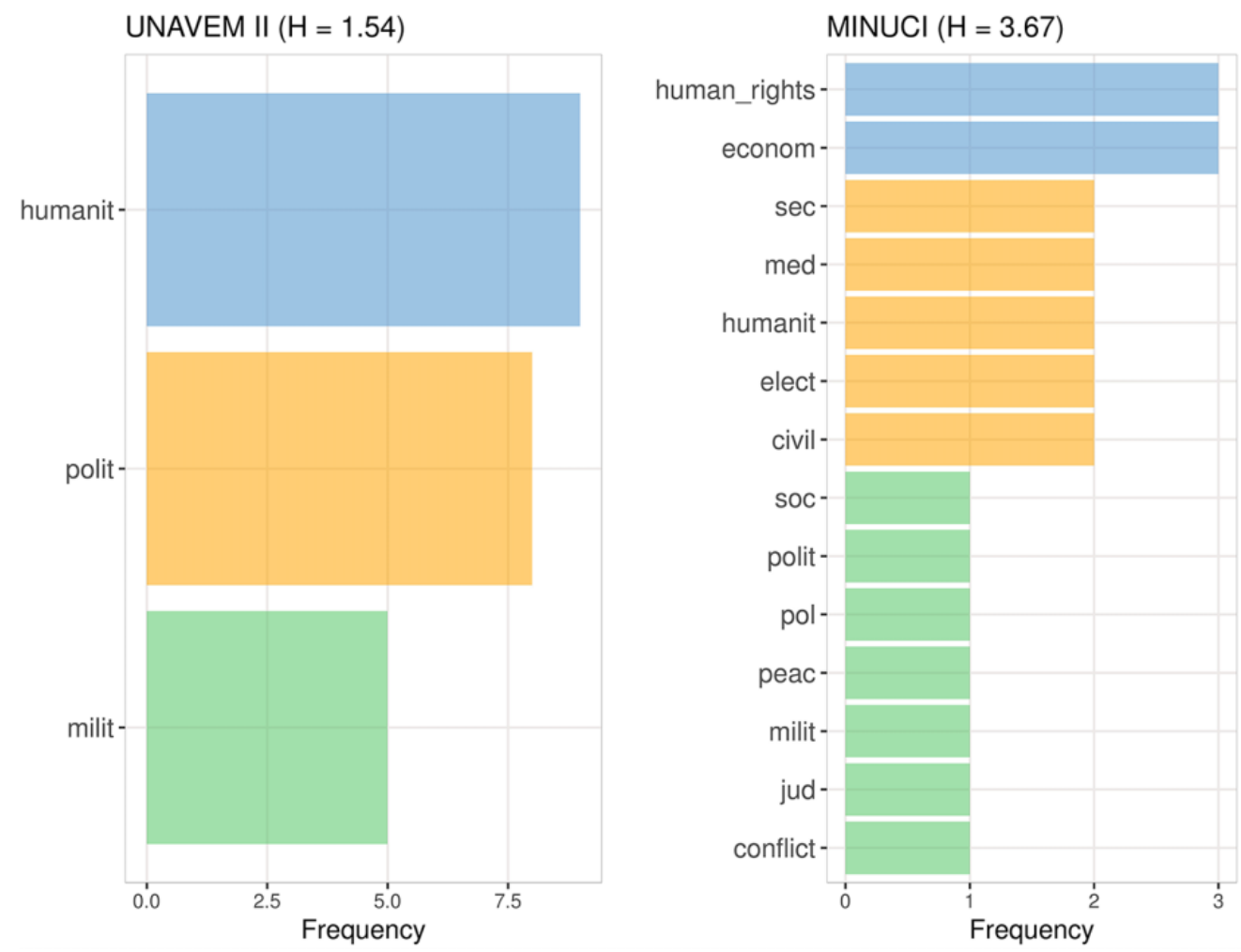

Figure 7. Comparing domains of activity in UNAVEM II and MINUCI

number of domains is larger, and includes elections, police and judiciary, most of which were in fact part of MINUCI's mandate. But as with UNAVEM II, MINUCI reports also substantially focus on a nonmandated task, namely the economic situation (without a mandate to support economic recovery). This example illustrates two key dynamics. First, UNSG reports do not strictly follow UNSC mandated tasks. They may report on issues that are not part of the mandate or may not report on tasks blue helmets are expected to carry out.

Second, Figures 6 and 7 show that missions vary in terms of attention they pay to different domains of activities, and it is possible to classify them accordingly.

Besides these static comparisons, we select UNMIK to explore how the domain of activities changes in the life-cycle of PKOs. Figure 8 plots all activity domains of UNMIK on the vertical axis, and the month of reporting on the horizontal axis. Notice each column is a reporting-month, not a deployment-month. Orange tiles indicate that a certain domain is present in reports released in a given month. Fully blue columns indicate reports with no informative titles (see Online appendix A.4).
In the first five years of deployment, UNMIK activities involved economic, humanitarian and reconstruction tasks. Overall, UNMIK performed activities in a wide range of domains in the first phase of deployment (see Figure 8, red rectangle). This ambitious approach changed later on, when the mission apparently specialized in fewer domains (see Figure 8, blue rectangle), some of which were completely new to the mission, such as religion, culture and women. Other domains that were already present before to some extent, also became steadily prominent, such as rule of law, returnees and human rights.

The evolution of UNMIK suggests that the mission went through some important adjustments. In the first five years, the domains of activities kept changing and the mission seemed to stabilize only in the second half of its (ongoing) life-cycle. As highlighted by Di Salvatore \& Ruggeri (2017: 20), '[s]ystematic identification of stages in peacekeeping operations would provide interesting insights into which main phases the most successful missions go through'. PKOC allows examination of whether similar patterns, or 'phases', exist in other PKOs, and to quantify their dynamics in a way that captures variation across missions, which currently remain bundled up in dichotomies that rely on mandates rather than actual activities. 


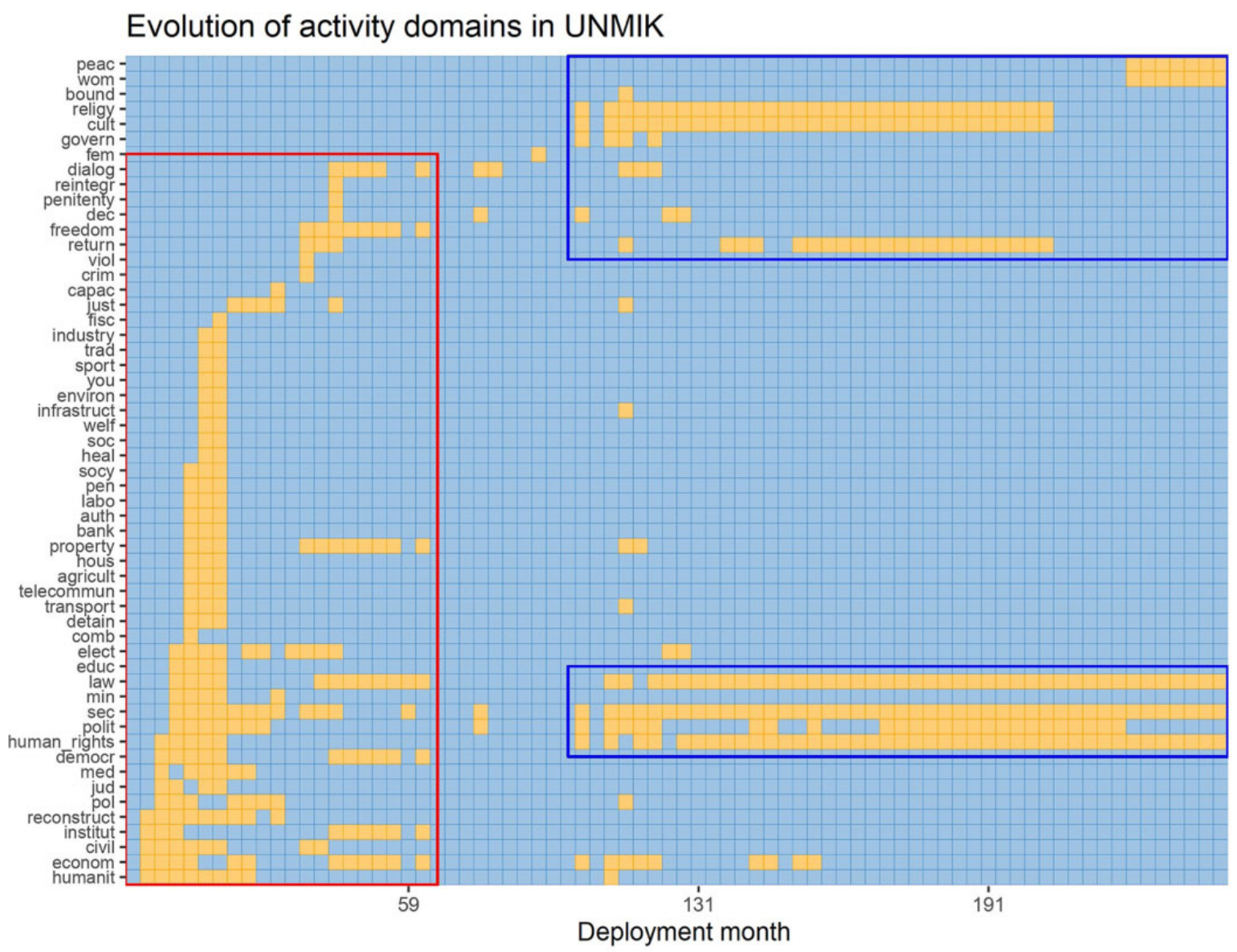

Figure 8. Evolution of UNMIK's multidimensionality

\section{Conclusion}

In this article, we introduced PKOC as the first step toward the use of text-as-data on UNSG reports on peacekeeping. The combination of PKOC with other ongoing projects on corpora of text from other UN institutions, such as the General Assembly's speeches (Baturo, Dasandi \& Mikhaylov, 2017) and UNSC debates (Schoenfeld et al., 2019), represents a fertile ground for future research that aims at understanding UN dynamics among and within UN key institutions. Indeed, given the strategic and political orientation of UNSG reporting, we believe that this is where the main contribution of PKOC lies. Analysing UNSG reports as objective reporting of PKOs should not overlook that the intent of these documents is not to accurately document what blue helmets do, and that the risk of reporting bias is not negligible. Hence, PKOC can support research at different stages, from preliminary exploration to measurement of key variables and more advanced content analysis. We discussed how its indexing structure increases the flexibility of interrogation and data extraction, making it possible to query the corpus by mission, deployment period, month/year, host country and report code. Furthermore, we discussed how the characteristics of the three corpus' formats (plain, reduced and tagged) enhance the flexibility of researchers on the analytical side by widening the range of tools and perspectives by which peacekeeping can be studied.

\section{Replication data}

The dataset, codebook, and do-files for the empirical analysis in this article, along with the Online appendix, are available at https://www.prio.org/jpr/datasets/. All analyses were conducted using R (3.5) and Python (3.7).

\section{Acknowledgements}

We thank Daniel Lozano Rojas for his excellent research assistance.

\section{ORCID iD}

Jessica Di Salvatore (D) https://orcid.org/0000-00017654-9794 


\section{References}

Baker, Paul (2006) Using Corpora in Discourse Analysis. London: Continuum.

Baturo, Alexander; Niheer Dasandi \& Slava J Mikhaylov (2017) Understanding state preferences with text as data: Introducing the UN General Debate corpus. Research o Politics 4(2): 1-9.

Benoit, Kenneth; Drew Conway, Benjamin E Lauderdale, Michael Laver \& Slava J Mikhaylov (2016) Crowd-sourced text analysis: Reproducible and agile production of political data. American Political Science Review 110(2): 278-295.

Benoit, Kenneth; Kohei Watanabe, Haiyan Wang, Paul Nulty, Adam Obeng, Stefan Müller \& Akitaka Matsuo (2018) Quanteda: An R package for the quantitative analysis of textual data. Journal of Open Source Software 3(30): 774 (https://quanteda.io).

Bove, Vincenzo; Chiara Ruffa \& Andrea Ruggeri (2020) Composing Peace: Mission Composition in UN Peacekeeping. Oxford: Oxford University Press.

Boydstun, Amber E; Shaun Bevan \& Herschel F Thomas III (2014) The importance of attention diversity and how to measure it. Policy Studies Journal 42(2): 173-196.

Cil, Deniz; Hanne Fjelde, Lisa Hultman \& Desirée Nilsson (2019) Mapping blue helmets: Introducing the Geocoded Peacekeeping Operations (Geo-PKO) dataset. Journal of Peace Research 57(2): 360-370.

Clayton, Govinda; Jacob Kathman, Kyle Beardsley, Theodora-Ismene Gizelis, Louise Olsson, Vincenzo Bove, Andrea Ruggeri, Reemco Zwetsloot, Jair van der Lijn \& Timo Smitothers (2017) The known knowns and known unknowns of peacekeeping data. International Peacekeeping 24(1): 1-62.

Di Salvatore, Jessica \& Andrea Ruggeri (2017) Effectiveness of peacekeeping operations. In: William $\mathrm{R}$ Thompson (ed.) The Oxford Encyclopedia of Empirical International Relations Theories. New York: Oxford University Press.

Dorussen, Han \& Theodora-Ismene Gizelis (2013) Into the lion's den: Local responses to UN peacekeeping. Journal of Peace Research 50(6): 691-706.

Dorussen, Han \& Andrea Ruggeri (2007) Introducing PKOLED: A peacekeeping operations location and event dataset. In: Conference on Disaggregating the Study of Civil War and Transnational Violence, University of Essex, 24-25 November (unpublished).

Fortna, Virginia Page \& Lise Morjé Howard (2008) Pitfalls and prospects in the peacekeeping literature. Annual Review of Political Science 11: 283-301.
Grimmer, Justin \& Brandon M Stewart (2013) Text as data: The promise and pitfalls of automatic content analysis methods for political texts. Political Analysis 21(3): 267-297.

Hultman, Lisa (2013) UN peace operations and protection of civilians: Cheap talk or norm implementation? Journal of Peace Research 50(1): 59-73.

Kjeksrud, Stian (2019) Using force to protect civilians. (Replication Data available at: https://doi.org/10.18710/ FZAVCN).

Manning, Christpher; Prabhakar Raghavan \& Hinrich Schutze (2008) Introduction To Information Retrieval. Cambridge: Cambridge University Press.

Manning, Christopher; Mihai Surdeanu, John Bauer, Jenny Finkel, Steven Bethard \& David McCloskye (2014) The Stanford CoreNLP natural language processing toolkit. In: Proceedings of 52nd Annual Meeting of the Association for Computational Linguistics: System Demonstrations (held June 2014 at ACL). Baltimore, MD: Association for Computational Linguistics, 55-60 (https://www.aclweb.org/ anthology/P14-5).

Munger, Kevin; Richard Bonneau, Jonathan Nagler \& Joshua A Tucker (2019) Elites tweet to get feet off the streets: Measuring regime social media strategies during protest. Political Science Research and Methods 7(4): 815-834.

Schoenfeld, Mirco; Steffen Eckhard, Ronny Patz \& Hilde van Meegdenburg (2019) The UN Security Council Debates. (Data available at: https://doi.org/10.7910/DVN/ KGVSYH).

Smidt, Hannah (forthcoming) Keeping electoral peace? Activities of United Nations peacekeeping operations and their effects on election-related violence. Conflict Management and Peace Science. (https://doi.org/10.1177/073 8894220960041).

UNSC (2009) Resolution 1894. S/RES/1894.

ELIO AMICARELLI, b. 1987, MSc Applied Statistics (University of St Andrews, 2015) and MSc Conflict Resolution (University of Essex, 2013); Data Science Solutions Team Leader at NTT DATA (2019- ); areas of expertise: causal inference, experimentation, machine learning.

JESSICA DI SALVATORE, b. 1988, PhD in Political Science (University of Amsterdam, 2017); Associate Professor at the University of Warwick (2020- ); current research interests: peacekeeping operations and political violence. 